\title{
All-Organic Waveguide Sensor for Volatile Solvent Sensing
}

\author{
Edgars NITISS*, Arturs BUNDULIS, Andrejs TOKMAKOVS, \\ Janis BUSENBERGS, and Martins RUTKIS
}

\author{
Institute of Solid State Physics, University of Latvia, Riga LV-1083, Latvia \\ ${ }^{*}$ Corresponding author: Edgars NITISS_Ｅ-mail: edgars.nitiss@cfi.lu.lv
}

\begin{abstract}
An all-organic Mach-Zehnder waveguide device for volatile solvent sensing is presented. Optical waveguide devices offer a great potential for various applications in sensing and communications due to multiple advantageous properties such as immunity to electromagnetic interference, high efficiency, and low cost and size. One of the most promising areas for applications of photonic systems would be real-time monitoring of various hazardous organic vapor concentrations harmful to human being. The optical waveguide volatile solvent sensor presented here comprises a novel organic material applied as a cladding on an SU-8 waveguide core and can be used for sensing of different vapors such as isopropanol, acetone, and water. It is shown that the reason for the chemical sensing in device is the absorption of vapor into the waveguide cladding which in turn changes the waveguide effective refractive index. The presented waveguide device has small footprint and high sensitivity of the mentioned solvent vapor, particularly that of water. The preparation steps of the device as well as the sensing characteristics are presented and discussed.
\end{abstract}

Keywords: Optical sensor; waveguide; organic materials; Mach-Zehnder interference

Citation: Edgars NITISS, Arturs BUNDULIS, Andrejs TOKMAKOVS, Janis BUSENBERGS, and Martins RUTKIS, "All-Organic Waveguide Sensor for Volatile Solvent Sensing," Photonic Sensors, 2019, 9(4): 356-366.

\section{Introduction}

Investigations into sensors for volatile organic compound detection have grown greatly and are motivated by the number of applications where they can be used, such as in food or chemical industry, electronic noses, or safety concerning toxic ambient conditions. In the last couple of decades, the waveguide photonics has been increasingly used in the measurement technology. A promising area for applications of photonic systems would be sensing of volatile solvents that are highly flammable, which has high reactivity risk and hazardous to human being and ecosystems in chemical manufacturing, civil engineering, and elsewhere [1]. The advantages of optical waveguide sensors are that they are immune to electromagnetic interference, they can be used for remote monitoring, and they are small and have a low weight.

Several different physical effects are employed in gas and organic compound sensing in optical devices [2]. The gas concentration is calculated from the light propagation loss in the waveguide by employing absorption based sensing [3-6]. Here, typical waveguide is operated in the mid-infrared range in which absorption peaks for a wide variety of trace gases are located. Such devices require fine optimization as well as expensive light sources and detectors. Another used approach for sensing of various organic molecules in waveguide devices is

Received: 20 September 2018 / Revised: 27 February 2019

(C) The Author(s) 2019. This article is published with open access at Springerlink.com DOI: $10.1007 / \mathrm{s} 13320-019-0543-\mathrm{Z}$

Article type: Regular 
the Raman spectroscopy [7]. The greatest advantage of such an approach is that it allows identification and quantification of molecules near the waveguide. Unfortunately, due to low Raman scattering cross-sections, the signals are typically weak and require using long optical waveguides.

A widely used effect in chemical sensing is the detection of change of refractive index caused by adsorption onto or absorption into materials [8]. Such refractive index changes cause resonance shifts in devices such as whispering gallery mode resonators [9-11], plasmonic devices [12], hetero-core [13] and hollow-core fiber sensors [14], and waveguide Mach-Zehnder interferometric (MZI) devices [15-18]. In all of the previously mentioned examples, the sensitivity is determined not only by the device design, but also by the employed materials. For applications in gas absorption, several different types of materials have been proposed in literatures, such as porous $\mathrm{SiO}_{2}$ or $\mathrm{TiO}_{2}$ glasses [19-21] and polymers [22-24].

In this contribution, we present an all-organic MZI waveguide device comprising novel organic vapor sensing organic material used as a cladding on SU-8 waveguide core. The beneficial properties of similar all-organic waveguide devices include the low cost of production, high sensitivity, and wide spectral bandwidth in the visible spectral range in which cheap detectors can be used, as well as the possibility to prepare the device on a mechanically flexible substrate $[25,26]$ which is relevant in applications such as wearable photonics [27]. For the developed sensor testing purposes, we have measured its sensitivity to isopropanol (IPA), acetonitrile (AN), dimethylformamide (DMF), and water and acetone vapors. The used solvent vapors are entitled to have different hazards, such as high flammability and influence on human health. The IPA and acetone belong to a group of chemicals that are highly flammable, while human exposure to AN and DMF can cause digestive and nervous system effects. The sensing within the presented optical device is enabled by the properties of used organic material in the cladding. It is porous and thus it may absorb the volatile solvents which in turn change their effective refractive index that can be sensed by the MZI. Yet we also show that using organic materials in the device brings drawbacks such as reduction of sensors sensitivity if used with solvent vapor that dissolves the cladding or core materials. Here the preparation steps, operation principles, sensitivity, waveguide thermo-optic coefficient, and other parameters of developed sensor are discussed.

\section{Experimental setup}

\subsection{Sensor preparation}

In advance to sensor preparation, we have carefully considered its design. The MZI sensor would be tested using a He-Ne laser operating at $632.8 \mathrm{~nm}$ thus waveguide engineering is done at this wavelength. The design of all-organic MZI waveguide sensor is illustrated in Fig. 1(a) where the sensor top-view as well as the employed waveguide cross-section is shown. The cross-section of the waveguide also includes the calculated mode profile according to parameters discussed below.

The waveguide MZI core is made of SU-8 negative-tone epoxy. One of the MZI arms is purposely made longer by an excess length of $L=$ $8 \mathrm{~mm}$. Such asymmetry enables refractive index variation sensitivity in the device which will be shown later. In top-view in Fig. 1(a), we also display the introduced tapers of $10-\mu \mathrm{m}$ width at the input of the device in such way that the light coupling efficiency can be increased to the best of our knowledge. The SU-8 resist is prepared by diluting GM-1075 SU-8 from Gerseltec in gamma-butyrolactone (GBL) in an 1:1 mass ratio. Then the prepared solution is spin-coated on a soda lime glass slide at $3000 \mathrm{rpm}$ for $60 \mathrm{~s}$. Afterwards, an MZI waveguide core structure is exposed in SU-8 using a laser writer Heidelberg $\mu \mathrm{PG} 101$ operating at $375 \mathrm{~nm}$ and developed. 

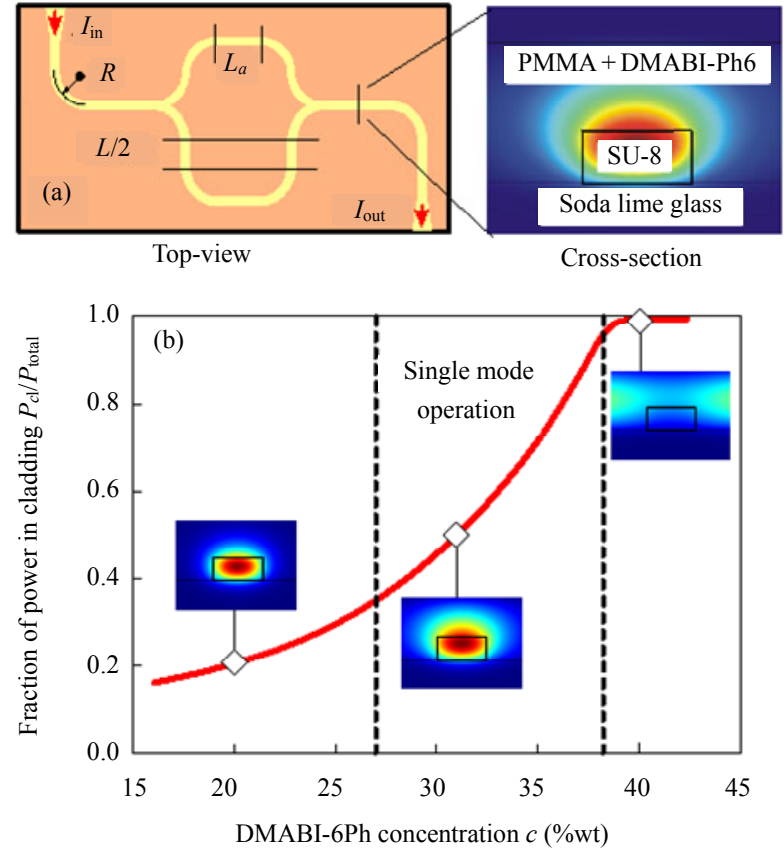

Fig. 1 Waveguide sensor device design and optical mode simulations: (a) the top-view and cross-section of the waveguide MZI, where $I_{\text {in }}$ and $I_{\text {out }}$ are the light input and output intensities, respectively; $I_{1}$ and $I_{2}$ are the light intensities in the MZI arms, $R=500 \mu \mathrm{m}$ is the bend radius of the waveguide, and $L=8 \mathrm{~mm}$ is the MZI arm excess length. The waveguide core is made of SU-8 and has the width of $1.5 \mu \mathrm{m}$ and the height of $0.7 \mu \mathrm{m}$. The cladding is a $1.2-\mu \mathrm{m}$-thick organic glass PMMA+DMABI-Ph6. The cross-section image also holds the calculated mode profile and (b) fraction of mode power in the cladding as a function of DMABI-Ph6 concentration $c$. Single mode operation is ensured when DMABI-Ph6 concentration is in the range from $27 \mathrm{wt} \%$ to $38 \mathrm{wt} \%$.

For proper operation of MZI sensor, it is essential to make the waveguide to operate in a single mode regime to avoid mode interference at the output. Typically, the single mode operation is reached by reducing the dimensions of the waveguide core. After series of experiments, we find that the optimal dimensions of waveguide core are $1.5 \mu \mathrm{m}$ in width and $0.7 \mu \mathrm{m}$ in thickness. Narrower waveguides are hard to reproduce with the available optical lithography workflow, and they become exceedingly fragile. The thickness of the waveguides is limited to around $0.7 \mu \mathrm{m}$ by the spin-coating technique and properties of the resist solution [28]. The refractive index of SU-8 is measured to be 1.589 using the m-line technique. We have used COMSOL Multphysics Wave Optics
Module to calculate the effective refractive indexes of modes in the waveguides. The simulations show that SU-8 waveguide with the mentioned dimensions and refractive index can support multiple modes. It is also calculated that in order to ensure that only a single optical mode can propagate through the waveguide MZI, it is necessary to apply a cladding which has the refractive index in the range from 1.559 to that of SU-8. If cladding with such a refractive index is added on the core, the second mode and higher order modes would be located in the cladding and would not be guided through the bends of MZI. The cladding would also have to operate as the gas sensing material. A good candidate for the mentioned application would be the poly(methyl methacrylate) (PMMA) polymer which has previously been reported to be used in gas sensing applications [29, 30]. PMMA refractive index is lower than that of SU-8, and it can be tuned by mixing it with a chromophore [31]. We use a 2-(4-(bis(5,5,5-triphenylpentyl)amino)benzylidene)1H-indene-1,3(2H)-dione (DMABI-Ph6) [32] as the chromophore for refractive index tuning in PMMA. The refractive index of PMMA+DMABI-Ph6 mix grows linearly with an increase of chromophore concentration $c$ expressed in wt $\%$ as DMABI-Ph6 and the PMMA + DMABI-Ph6 mass ratio. The refractive index $n$ of cladding as a function of concentration $c$ is experimentally measured to be $n=$ $2.67 \mathrm{e}-3 c+1.487$. According to previously discussed requirements, the waveguide would operate in a single mode regime if $c$ is in the range from $27 \mathrm{wt} \%$ to $38 \mathrm{wt} \%$. Figure 1 (b) shows the fraction of mode power in the cladding as a function of DMABI-Ph6 concentration. Here the fractions of mode power in the cladding is expressed as the ratio of power in the cladding $P_{c 1}$ and the total mode power $P_{\text {total }}$. The insets of Fig. 1(b) also show the calculated mode profiles. It is expected to have higher sensitivity when the mode is weakly confined. Unfortunately, the light propagation loss in the bends would also be higher for the weakly confined mode. To leverage 
this trade-off, the DMABI-Ph6 concentration is selected to be at around $32 \mathrm{wt} \%$ when half of the mode power is in the PMMA+DMABI-Ph6, and yet it should not leak out significantly while going through the bends. The prepared PMMA + DMABI-Ph6 organic glass is spin-coated at $2000 \mathrm{rpm}$ on the SU-8 waveguides from a chloroform solution. The thickness of the cladding is around $1.2 \mu \mathrm{m}$. Afterwards, the lower side of the substrate is cut by a diamond saw and the sample is broken along the cut line. The light can be coupled into the waveguide through the broken waveguide facet without any additional polishing or preparation steps.

The device sensitivity is enabled by the fact that the MZI is prepared asymmetrically. Any changes in the refractive index will cause variation in phase difference $\Delta \varphi$ in the MZI arms. This in turn will be transferred to light output intensity variations according to the two beam interference formula where output intensity $I_{\text {out }}$ is a function of $\cos (\Delta \varphi)$. The induced phase difference can be written as

$$
\Delta \varphi=\frac{2 \pi L}{\lambda} \Delta n_{\text {eff }}
$$

where $\Delta n_{\text {eff }}$ is the effective refractive index change of waveguide mode, $\lambda$ is the light wavelength, and $L$ is the MZI single arm excess length. The change of effective refractive index due to an external interaction $P$ can be calculated as

$$
\Delta n_{\mathrm{eff}}=\frac{\partial n_{\mathrm{eff}}}{\partial n} \frac{\partial n}{\partial P} \Delta P
$$

where $P$ is the physical parameter that is varied, for example temperature $T$, solvent concentration $C$ in the atmosphere or other. For the employed waveguides, we calculate the $\partial n_{\text {eff }} / \partial n$ to be around 0.3 [33].

\subsection{Sensor testing setup}

The sensor testing setup is illustrated in Fig. 2. We use He-Ne $632.8 \mathrm{~nm}$ laser, two mirrors, and a $20 \times$ microscope objective to excite the optical mode in the waveguide MZI sensor through the waveguide facet. We have also tried light coupling from a lensed fiber, however, with such coupling the sensor is more sensitive to mechanical noise and therefore is abandoned.

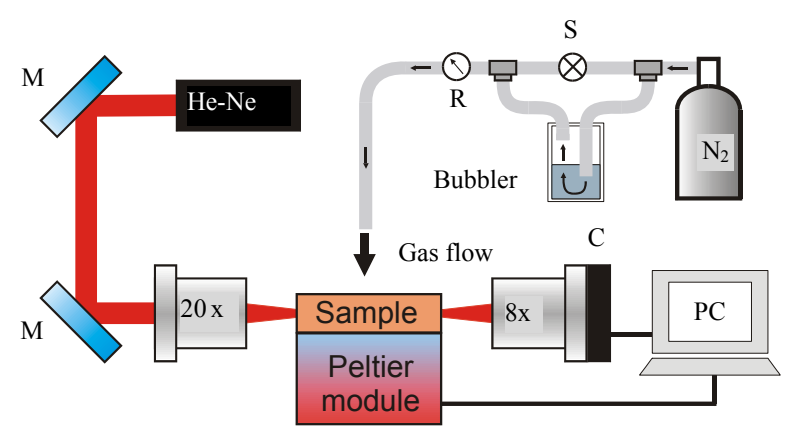

Fig. 2 Schematic illustration of waveguide device testing scheme. From a He-Ne laser the light is coupled into the Peltier module temperature stabilized sample using two mirrors $\mathrm{M}$ and a microscope objective, the output light intensity is collected on a camera $\mathrm{C}$. The $\mathrm{N}_{2}$ gas can be pumped through a bubbler with solvent or delivered directly to the sample depending on the switch $\mathrm{S}$ position. The gas flow is monitored by a rotameter $\mathrm{R}$.

The sample is glued with a heat conducting cobalt paste on a Peltier module for temperature stabilization and MZI device tuning when necessary. The temperature stability of the sample is controlled by a PID controller (Arroyo Instruments TECPak 585). At the output of MZI, the light is coupled on a CCD camera (Thorlabs DCC1545M) using $8 \times$ microscope objective. During the measurements, the light output intensity is retrieved using a MATLAB code from the camera images recorded at $100 \mathrm{fps}$. In our setup (shown in Fig. 2.) for testing the sensor response to organic vapor, we use an $\mathrm{N}_{2}$ as a carrier gas. $\mathrm{N}_{2}$ is selected as a chemically neutral gas in order to guarantee repeatable operation conditions throughout the sensor characterization process. A bubbler is used to mix the $\mathrm{N}_{2}$ with the solvent vapor. The response time and phase change amplitude of the sensor are measured by alternating the gas stream flowing on the sample between the pure $\mathrm{N}_{2}$ stream (OFF state) and the mixed organic vapor $/ \mathrm{N}_{2}$ stream (ON state). We use a heat exchanger to ensure that the gas flow temperature is the same as room temperature to avoid sample cooling or heating. In the gas delivery system, we use a rotameter to control the gas flow onto to the sample. 
The gas flow is set to be $25 \mathrm{ml} / \mathrm{s}$. The solvent concentration in the $\mathrm{N}_{2}$ gas during mixing is estimated by measuring the weight loss of the solvent in time, which is then used to calculate the consumed gas volume and divided by the overall consumed volume of solvent $/ \mathrm{N}_{2}$ mix on the sample. The described approach on mixing and delivering the gas mix to sample has some important advantages. It is very easy to implement for measuring the response of a free-space coupled optical chip. Moreover, the response time of such a system is short, and thus the sensor detection speed can be evaluated. A major drawback of the approach is that the bubbler system may show fluctuations in test gas generation as reported elsewhere [34]. As shown below, the main cause of error while measuring the sensor response is due to uncertainty of solvent concentration provided by the vapor delivery system.

\section{Results and discussion}

\subsection{Thermal tuning}

Initially, the thermal tunability of the device is tested. The sample temperature is increased linearly in time at rate of $0.1 \%$, and simultaneously the light output intensity is measured. The obtained light output intensity is represented by the blue points in Fig. 3. Using the thermal tuning measurements, we are able to determine the phase change in the MZI as a function of intensity change which is particularly important for performing sensing measurements.

As evident from Fig. 3, the light output intensity changes in an oscillating manner with maxima $I_{\max }$ and minima $I_{\min }$ corresponding to constructive and destructive interference, and are indicated with red points. The amplitude of the signal decreases due to thermal detuning indicated by the orange line. This is due to the change in the sample position relative to the coupling spot caused by thermal expansion of substrate, Peltier element, and cobalt paste. From the extreme points in the graph, the phase change as a function of temperature can be calculated. The results are shown in the inset of Fig. 3. By using phase difference as a function of temperature (see inset) and (2), the thermo-optic coefficient $\partial n / \partial T$ is calculated to be around $-3.31 \mathrm{e}-4(1 / \mathrm{K})$. Since we cannot determine the sign of thermo-optic coefficient from our measurements, we assume that it is negative. The thermo-optic coefficients in polymers are typically negative due to a decrease in polymer density with an increase in temperature [35]. The calculated thermo-optic coefficient of SU-8 and PMMA+DMABI-Ph6 waveguide is close to that of pure SU-8 which has been reported to be $-1.1 \mathrm{e}-4$ $(1 / \mathrm{K})[36,37]$. With high thermo-optic coefficients of described waveguide materials, they might have application in thermo-optical switches [37-39]. In further measurements of sensor sensitivity to volatile solvent vapor, the sensor temperature is kept constant at room temperature using a PID controller.

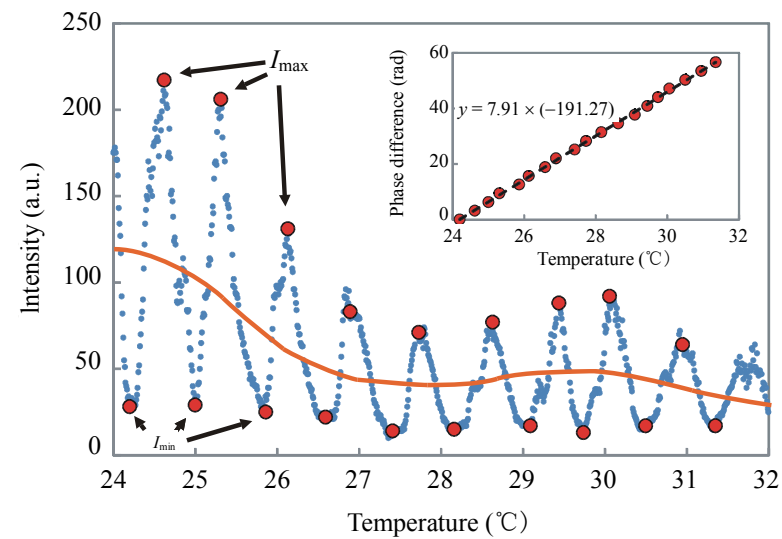

Fig. 3 Output light intensity $I_{\text {out }}$ as a function of sample temperature is indicated by blue points. The red points indicate the maxima and minima of constructive or destructive interference, respectively. The orange line indicates the optical coupling detuning due to waveguide position drift during the temperature sweep. The inset shows the calculated phase difference in radians as a function of temperature.

\subsection{Volatile solvent vapor sensing}

The sensitivity of sensor is tested to various organic vapor-IPA, AN, DMF, water, and acetone. Such solvents are selected due to their different properties, e.g., molecule size, polarity, refractive index, and the sensitivity to which could provide an 
insight into understanding the sensing mechanism of the sensor.

The polymer that is used as cladding in the sensor has poor solubility in most used solvents except for acetone in which the PMMA-DMABI-Ph6 dissolves very well. In Fig. 4, as an example of sensor operation, the gas flow induced phase shift in the MZI sensor is shown.

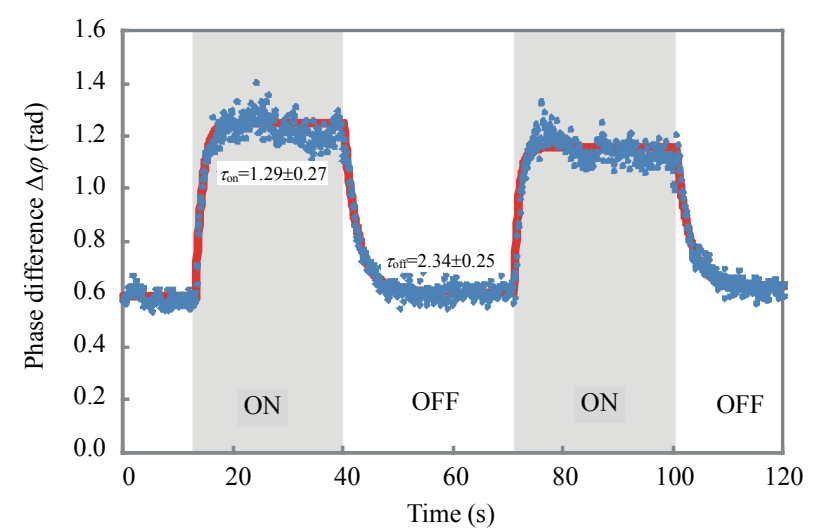

Fig. 4 Measured sensor comprising a waveguide with $1.2 \mu \mathrm{m}$ thick organic glass PMMA + DMABI-Ph6 cladding response to the gas stream flowing on the sample in time, where the OFF state corresponds to pure $\mathrm{N}_{2}$ stream, while the $\mathrm{ON}$ state to the mixed IPA $/ \mathrm{N}_{2}$ stream.

Here the ON and OFF states correspond to pure $\mathrm{N}_{2}$ stream and mixed IPA/ $\mathrm{N}_{2}$ stream, respectively. By using single exponent decay function, the $\mathrm{ON}$ and OFF switching times $\tau_{\text {on }}$ and $\tau_{\text {off }}$ are determined. The sensitivities of MZI waveguide device to different organic solvent vapors are summarized in Table 1 . Here the phase changes $\Delta \varphi$ induced by solvent vapor volume concentration $C$ along with calculated $\mathrm{ON}$ and OFF switching times are displayed. In Table 1, we have also included the measurement errors. The main cause of the sensitivity measurement error is due to imprecisions of solvent vapor concentration $C$ measurement.

Due to measurement noise during switching, we are not able to determine the switching times for DMF and water cases. From Table 1, it can be noticed that the sensitivity of the device varies within three orders of magnitude, and the sensor is least sensitive to acetone and has highest sensitivity to water vapor. Since the sensitivity of water in the gas is extremely higher than that of other solvents, we are concerned with the water content, which could be adsorbed from the air in other solvents, would be responsible for sensitivity levels of those gasses. The measured water content in AN is below $0.1 \%$ while in IPA, DMF, and acetone, the water content is below $0.05 \%$. Due to such low levels of water content in those solvents, it is safe to say that the measured sensitivity is almost entirely determined by the solvent properties.

Table 1 Experimental parameters and results of volatile solvent sensing using a waveguide with $1.2 \mu \mathrm{m}$ thick organic glass PMMA + DMABI-Ph6 cladding. Here $C$ is the organic solvent gas volume concentration in $\mathrm{N}_{2}, \Delta \varphi$ is the resulting phase change, $\tau_{\text {on }}$ and $\tau_{\text {off }}$ are the ON and OFF switching times, and $(\Delta \varphi / C) \times 100$ is the calculated sensitivity of MZI waveguide device to vapor of different organic solvents.

\begin{tabular}{ccccc}
\hline & $C(\%)$ & $\Delta \varphi(\mathrm{rad})$ & $\begin{array}{c}\tau_{\text {on }}(\mathrm{s}) \\
\tau_{\text {off }}(\mathrm{s})\end{array}$ & $\begin{array}{c}(\Delta \varphi / C) \times 100 \\
(\mathrm{rad} / \%)\end{array}$ \\
\hline IPA & $3.06 \pm 0.10$ & $0.66 \pm 0.07$ & $\begin{array}{l}1.29 \pm 0.27 \\
2.34 \pm 0.25\end{array}$ & $21.6 \pm 2.35$ \\
& & & $1.39 \pm 0.16$ & $225 \pm 24$ \\
AN & $2.16 \pm 0.11$ & $4.86 \pm 0.47$ & $1.82 \pm 0.21$ & \\
& & & - & $153 \pm 78$ \\
DMF & $0.46 \pm 0.19$ & $0.70 \pm 0.20$ & - & \\
& & & - & $3469 \pm 606$ \\
Water & $0.76 \pm 0.13$ & $26.51 \pm 0.45$ & $0.46 \pm 0.10$ & \\
& & & $0.81 \pm 0.07$ & $4.6 \pm 0.84$ \\
\hline
\end{tabular}

Several phenomena could account for the sensitivity of sensor to solvent vapor. The sensing could be due to the adsorption of solvent molecules onto surface which induces swelling and hence tension in the waveguide [40-42]. The tension may cause refractive index change due to stress-optical effect. In such a case, the waveguide sensor should have sensitivity dependence on the cladding thickness, which should be less sensitive for thicker claddings. We therefore make an MZI waveguide sensor with $4.4 \mu \mathrm{m}$ thick organic glass PMMA + DMABI-Ph6 cladding. The sensitivities and time constants measured in response to water and IPA vapor are the same within the margin of measurement error as for the sample with $1.2 \mu \mathrm{m}$ thick cladding. Thus we propose that the solvent penetration into the waveguide MZI is responsible for causing output intensity variations of the sensor. 
As a simple approximation, we propose that the refractive index of the polymer could vary due to absorption of molecules within the free volume and pores of PMMA-DMABI-Ph6 [29] or SU-8 [43]. To distinguish whether the effect is due to solvent absorption in the cladding or core of the waveguide, we have measured the sensitivity of the sensor after heating the sample at $120{ }^{\circ} \mathrm{C}$ for $30 \mathrm{~min}$. This temperature is above the glass transition temperature of the cladding which is at $80^{\circ} \mathrm{C}$ [31], and below the glass transition temperature of the SU-8. In Fig. 5, the normalized phase change as a function of time for sensor before and after heating is shown. Apparently, the phase change of the preheated sensor at the same influence has reduced by around $60 \%$, which implies that the cladding is responsible for sensing the presence of volatile solvent vapor.

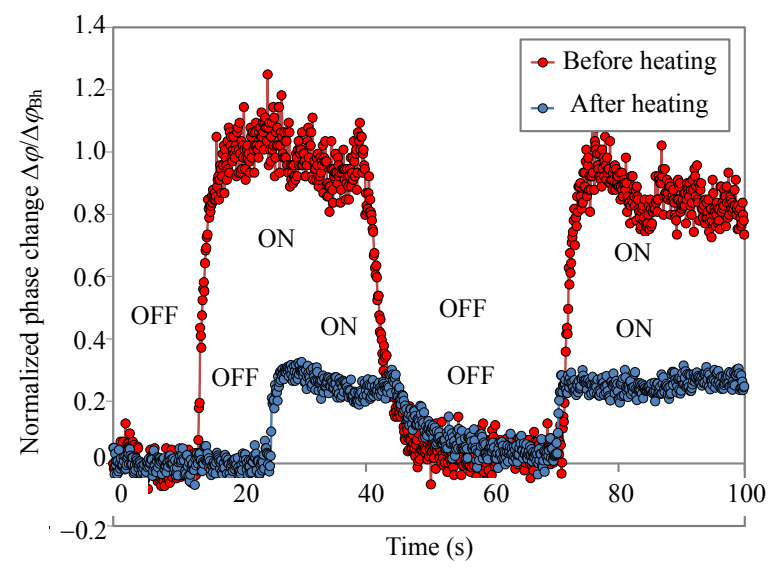

Fig. 5 Normalized phase change as a function of time for sensor before and after heating at $120^{\circ} \mathrm{C}$ for $30 \mathrm{~min}$. The $\Delta \varphi_{\mathrm{Bh}}$ is the phase change amplitude of nonpreheated sample at the influence of IPA / $\mathrm{N}_{2}$ stream. The sensor comprises a waveguide with $1.2 \mu \mathrm{m}$ thick organic glass PMMA+DMABI-Ph6 cladding. Here the OFF state corresponds to pure $\mathrm{N}_{2}$ stream, while the $\mathrm{ON}$ state to the mixed IPA/ $\mathrm{N}_{2}$ stream.

It is expected that the spin-coated PMMA+DMABI-Ph6 would be nano-porous since it is made of a highly volatile chloroform solution. The average free volume radius in PMMA has been measured to be just below $0.3 \mathrm{~nm}$ [44]. Thus during sensor operation, the free volume and pores would be filled with a vapor with larger refractive index than that of $\mathrm{N}_{2}$ subsequently increasing the refractive index of cladding and causing phase changes in the MZI. After subjecting the sample to solvent vapor $/ \mathrm{N}_{2}$ mix, the solvent would penetrate into the waveguide material until reaching an equilibrium solvent concentration.

Some studies of solvent molecule penetration in the bulk PMMA while kept saturated solvent vapor [29] or directly in solvent [45] contradict our observations in two aspects. Firstly, the switching time determined by vapor diffusion in our MZI waveguide sensor demonstrated here is in the range of seconds, while studies in bulk suggest diffusion rate to be at the order of $\mathrm{nm} / \mathrm{min}[29,45]$. Secondly, the equilibrium concentration for different solvents in PMMA measured by Ballenger et al. does not correlate with the demonstrated sensor sensitivity [29]. Yet another study in which PMMA is subjected to low concentration vapor supports our hypothesis on volume filling. Matsuguchi et al. demonstrated a capacitive humidity sensor in which gas sorption in micrometer thick PMMA films was shown to take place within seconds [30].

The solvent equilibrium concentration and diffusion rate in PMMA depend on multiple factors such as the nature of the solvent (poorly, moderately, or strongly hydrogen bonded), its solubility parameter, and size of molecules as identified in the PMMA bulk studies [29]. Yet previous studies in thin films have shown that the solvent penetration rates in PMMA are strongly dependent on the molecule size of solvents [45], which agrees well with the free volume model [46]. We observe that the experimentally switching time, which is proportional to the diffusion rate, correlates with the molecule size. As seen from Table 1, the sensor has the fastest response to water and AN. These solvents have small molecules. Yet the switching time is slower for acetone and IPA vapor which have slightly larger molecules than those of water and AN. Equilibrium concentration of solvent in the cladding could also depend on the molecule size. Assuming that there is a distribution of size of pores and free 
volume, the smaller molecules would be in larger concentrations than the larger ones in the cladding. This is also supported by the previous measurement of nonpreheated and preheated sensor sensitivity shown in Fig. 5. We attribute the sensitivity decrease of preheated sample to the PMMA + DMABI-Ph6 densification causing the reduction of free volume in the material.

In Fig. 6, we show the sensitivity of MZI waveguide device to vapor of different organic solvents as a function of solvent molecule radius $r$ calculated using spherical molecule approximation:

$$
r=\sqrt[3]{\frac{3 M}{4 \pi \rho N_{A}}}
$$

where $N_{A}$ is the Avogadro number, $M$ is the molar mass, and $\rho$ is the solvent density. The calculated molecule radius is of the same order as the measured free volume in PMMA [44]. As evident from Fig. 6, the sensitivity of the sensor reduces exponentially with an increase in the organic vapor molecule size for most of the solvents except IPA and DMF. Obviously, other parameters besides the molecule size could influence the solvent molecule adsorption process, but they will not be further discussed in this paper. These results support the hypothesis that the sensing is due to filling of pores in the polymer by the solvent molecules.

For practical applications, it is important to estimate the device sensitivity threshold. The sensitivity threshold can be estimated from the data in Table 1. If it is assumed that sensitivity threshold is around three times that of obtained error, then it is easy to show that, for example the sensitivity threshold for IPA vapor is around $0.01 \%$. This is of the same order as for commercially available inorganic $\mathrm{SnO}_{2}$ sensors in which the vapor concentration is related to the material resistivity change [47]. Yet, as seen from Table 1 and Fig. 6, from all of the used gases the sensor is most sensitive to water vapor. The $0.76 \%$ water vapor $/ \mathrm{N}_{2}$ mix, which is in relative humidity units, is approximate $24.3 \%$ rh caused phase changes of around $8.5 \pi$.

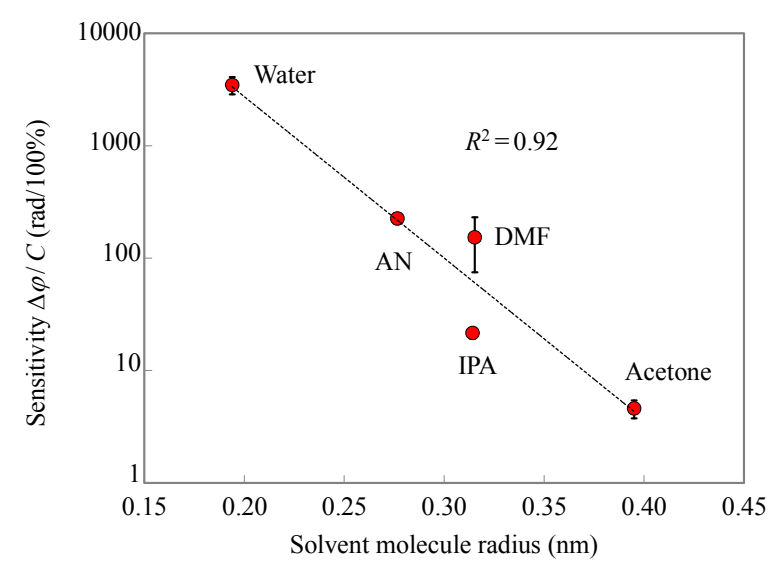

Fig. 6 Sensitivity of MZI waveguide sensor to vapor of different organic solvents as a function of solvent molecule radius. PMMA + DMABI-Ph6 cladding thickness is $1.2 \mu \mathrm{m}$.

The sensitivity threshold of demonstrated sensor can be significantly increased by reducing the measurement error. In this work, the main causes of measurement error are the thermal and light coupling stability as well as vapor delivery conditions. The direct vapor flow on the sample causes the relative error to be around $10 \%$ of the measured value. Clearly, the relative error would be much less if the sample is tested in a gas chamber, and the waveguide device is packaged.

\subsection{Aging and recovery measurements}

For measuring the aging of the sensor, it is subjected to a constant flow of solvent vapor $/ \mathrm{N}_{2}$ mix for several hours. Occasionally, the sensor sensitivity to that solvent vapor $/ \mathrm{N}_{2}$ mix is measured as described in Section 3.2. In Fig. 7, the measured phase differences $\Delta \varphi$ induced by acetone $/ \mathrm{N}_{2}$ and IPA $/ \mathrm{N}_{2}$ are displayed.

The IPA and acetone are selected as representatives of solvents in which the PMMA+ DMABI-Ph6 has poor and good solubilities, respectively. We find that the constant IPA $/ \mathrm{N}_{2}(C=$ $3.06 \%)$ and acetone $/ \mathrm{N}_{2}(C=13.29 \%)$ flow on the device reduces its sensitivity significantly. It can be seen that the device sensitivity reduces almost four-fold in just two hours. This must be due to the 
adsorption of the solvent in the cladding which results in lower porosity of material and thus lower response to atmosphere change. However, the sensor can "heal" if the solvent is allowed to evaporate from the cladding material. After drying of the sensor in air for 24 hours, the response returns to the previous condition (see Fig. 7).

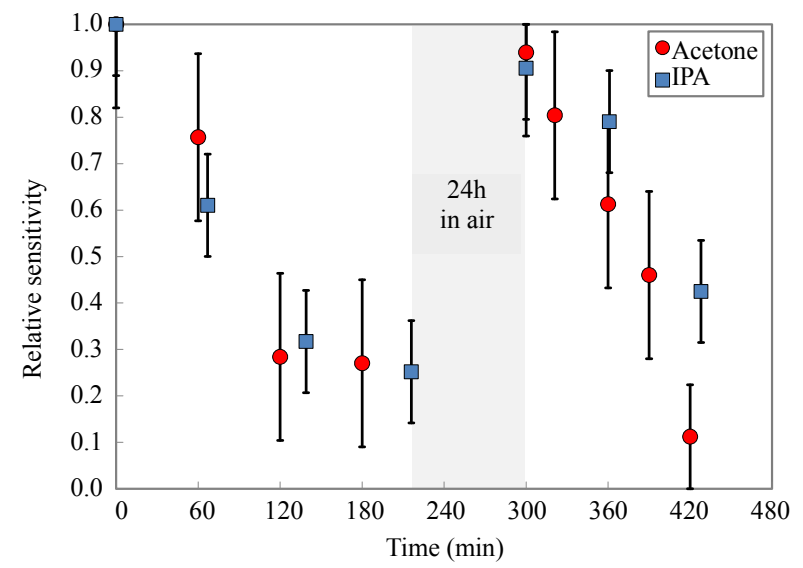

Fig. 7 Relative sensitivity of MZI waveguide device time when subjected to continuous acetone / $\mathrm{N}_{2}(C=13.29 \%)$ and IPA $/ \mathrm{N}_{2}$ flow $(C=3.06 \%)$ flow.

\section{Conclusions}

We have demonstrated the preparation steps and characterization results of an asymmetric all-organic waveguide MZI volatile solvent vapor sensor comprising SU-8 waveguide core and PMMA + DMABI-Ph6 cladding. By using thermal tuning enabled by a Peltier element, we are able to measure the thermo-optic coefficient which is $-3.31 \mathrm{e}-4(1 / \mathrm{K})$. Because the latter device is very sensitive to temperature variations, thermal stabilization is necessary for the device to be used in practical applications. The thermally stable MZI sensor is subjected to flow of various volatile solvent vapors. We demonstrate that the sensitivity of the device is due to filling the free volume and pores of the waveguide cladding with the volatile solvent vapor. It is shown that the sensitivity of the sensor reduces exponentially with an increase in the solvent vapor molecule size. Unfortunately, the sensor cannot be used for a long period to detect solvents in which the cladding material has good solubility. Due to intercalation of such solvent in the cladding, the material swelling takes place. As a result, it has lower porosity which in turn dramatically lowers the sensor response to atmosphere change.

\section{Acknowledgment}

This work was supported by ERDF 1.1.1.1 Activity Project Nr. 1.1.1.1/16/A/046 "Application assessment of novel organic materials by prototyping of photonic devices". We acknowledge Igors MIHAILOVS for valuable discussions.

Open Access This article is distributed under the terms of the Creative Commons Attribution 4.0 International License (http://creativecommons.org/licenses/by/4.0/), which permits unrestricted use, distribution, and reproduction in any medium, provided you give appropriate credit to the original author(s) and the source, provide a link to the Creative Commons license, and indicate if changes were made.

\section{References}

[1] S. Pandey, "Highly sensitive and selective chemiresistor gas/vapor sensors based on polyaniline nanocomposite: a comprehensive review," Journal of Science: Advanced Materials and Devices, 2016, 1(4): 431-453.

[2] O. S. Wolfbeis, "Fiber-optic chemical sensors and biosensors," Analytical Chemistry, 2006, 78(12): 3859-3874.

[3] M. A. Butt, S. N. Khonina, and N. L. Kazanskiy, "Silicon on silicon dioxide slot waveguide evanescent field gas absorption sensor," Journal of Modern Optics, 2018, 65(2): 174-178.

[4] R. Wang, A. Vasiliev, M. Muneeb, A. Malik, S. Sprengel, G. Boehm, et al., "III-V-on-silicon photonic integrated circuits for spectroscopic sensing in the 2-4 $\mu \mathrm{m}$ wavelength range," Sensors, 2017, 17(8): 1788-1-1788-21.

[5] M. A. Butt, S. A. Degtyarev, S. N. Khonina, and N. L. Kazanskiy, "An evanescent field absorption gas sensor at mid-IR $3.39 \mu \mathrm{m}$ wavelength," Journal of Modern Optics, 2017, 64(18): 1892-1897.

[6] M. A. Butt, S. N. Khonina, and N. L. Kazanskiy, "Modelling of Rib channel waveguides based on silicon-on-sapphire at $4.67 \mu \mathrm{m}$ wavelength for evanescent field gas absorption sensor," Optik, 2018, 168: 692-697.

[7] A. Dhakal, P. C. Wuytens, F. Peyskens, K. Jans, N. Le Thomas, and R. Baets, "Nanophotonic waveguide enhanced Raman spectroscopy of 
biological submonolayers," ACS Photonics, 2016, 3(11): 2141-2149.

[8] J. Milvich, D. Kohler, W. Freude, and C. Koos, "Surface sensing with integrated optical waveguides: a design guideline," Optics Express, 2018, 26(16), 19885-19906.

[9] M. R. Foreman, J. D. Swaim, and F. Vollmer, "Whispering gallery mode sensors," Advances in Optics and Photonics, 2015, 7(2): 168-240.

[10] A. R. Ali and C. M. Elias, "Ultra-sensitive optical resonator for organic solvents detection based on whispering gallery modes," Chemosensors, 2017, 5(2): 19-1-19-10.

[11] L. L. Páez, K. S. Carracedo, M. H. Rodríguez, I. R. Martín, T. Carmon, and L. L. Martin, "Liquid whispering-gallery-mode resonator as a humidity sensor," Optics Express, 2017, 25(2): 1165-1172.

[12] O. Hugon, P. Benech, and H. Gagnaire, "Surface plasmon chemical/biological sensor in integrated optics," Sensors and Actuators B: Chemical, 1998, 51(1): 316-320.

[13] Q. Wu, Y. Semenova, J. Mathew, P. F. Wang, and G. Farrell, "Humidity sensor based on a single-mode hetero-core fiber structure," Optics Letters, 2011, 36(10): 1752-1754.

[14] P. M. P. Gouvêa, P. Rugeland, M. S. P. Gomes, and W. Margulis, "Component and setup for insertion of gases in a hollow-core optical fiber sensor," $S P I E$, 2015, 9634: 96343D-1-96343D-4.

[15] S. Dante, D. Duval, B. Sepúlveda, A. B. G. Guerrero, J. R. Sendra, and L. M. Lechuga, "All-optical phase modulation for integrated interferometric biosensors," Optics Express, 2012, 20(7): 7195-7205.

[16] K. Misiakos, I. Raptis, E. Makarona, A. Botsialas, A. Salapatas, P. Oikonomou, et al., "All-silicon monolithic Mach-Zehnder interferometer as a refractive index and bio-chemical sensor," Optics Express, 2014, 22(22): 26803-26813.

[17] P. Dumais, C. L. Callender, J. P. Noad, and C. J. Ledderhof, "Integrated optical sensor using a liquid-core waveguide in a Mach-Zehnder interferometer," Optics Express, 2008, 16(22): 18164-18172.

[18] N. Fabricius, G. Gauglitz, and J. Ingenhoff, “A gas sensor based on an integrated optical Mach-Zehnder interferometer," Sensors Actuators B: Chemical, 1992, 7(1): 672-676.

[19] P. J. Skrdla, S. S. Saavedra, N. R. Armstrong, S. B. Mendes, and N. Peyghambarian, "Sol-Gel-based, planar waveguide sensor for water vapor," Analytical Chemistry, 1999, 71(7): 1332-1337.

[20] L. Yang, S. S. Saavedra, and N. R. Armstrong, "Sol-Gel-based, planar waveguide sensor for gaseous iodine," Analytical Chemistry, 1996, 86(11): 1834-1841.

[21] Z. Zhang, D. F. Lu, and Z. M. Qi, “Application of porous $\mathrm{TiO}_{2}$ thin films as wavelength-interrogated waveguide resonance sensors for bio/chemical detection," The Journal of Physical Chemistry C, 2012, 116(6): 3342-3348.

[22] R. Amberkar, Z. Gao, J. Park, D. B. Henthorn, and C. S. Kim, "Process development for waveguide chemical sensors with integrated polymeric sensitive layers," SPIE, 2008, 6886: 68860U-1-68860U-8.

[23] F. L. Alves, I. M. Raimundo, I. F. Gimenez, and O. L. Alves, "An organopalladium-PVC membrane for sulphur dioxide optical sensing," Sensors Actuators $B$ Chemical, 2005, 107(1): 47-52.

[24] A. Gastón, F. Pérez, and J. Sevilla, “Optical fiber relative-humidity sensor with polyvinyl alcohol film," Applied Optics, 2004, 43(21): 4127-4132.

[25] N. Zhao, G. Qian, X. C. Fu, L. J. Zhang, W. Hu, R. Z. $\mathrm{Li}$, et al., "Integrated optical displacement sensor based on asymmetric Mach-Zehnder interferometer chip," Optical Engineering, 2017, 56(2): 027109-1-027109-6.

[26] Y. Huang, G. T. Paloczi, J. K. S. Poon, and A. Yariv, "Demonstration of flexible freestanding all-polymer integrated optical ring resonator devices," Advanced Materials, 2004, 16(1): 44-48.

[27] M. Crawford, "Wearable technology is booming, powered by photonics," SPIE, 2016, DOI: 10.1117/2.2201606.01.

[28] E. Nitiss, J. Busenbergs, A. Tokmakovs, and M. Rutkis, "Preparation of an organic waveguide electro-optic modulator operating in the visible spectral range," Sensors Transducers, 2018, 225(9): 19-24.

[29] V. Ballenger, J. K. Commerçon, J. Verdu, and P. Tordjeman, "Interactions of solvents with poly (methyl methacrylate)," Polymer, 1997, 38(16): 4175-4184.

[30] M. Matsuguchi, Y. Sadaoka, Y. Sakai, T. Kuroiwa, and A. Ito, "A capacitive-type humidity sensor using cross-linked poly (methyl methacrylate) thin films," Journal of The Electrochemical Society, 1991, 138(6): 1862-1865.

[31] E. Nitiss, A. Tokmakovs, K. Pudzs, J. Busenbergs, and M. Rutkis, "All-organic electro-optic waveguide modulator comprising SU-8 and nonlinear optical polymer," Optics Express, 2017, 25(25): 31036-31044.

[32] K. Traskovskis, I. Mihailovs, A. Tokmakovs, V. Kokars, and M. Rutkis, "An improved molecular design of obtaining NLO active molecular glasses using triphenyl moieties as amorphous phase formation enhancers," SPIE, 2012, 8434: 84341P-1-84341P-8.

[33] E. Nitiss, "Evaluation of performance of a hybrid electro-optic directional coupler and a Mach-Zehnder switch," Journal of Nanophotonics, 
2017, 11(1): 016013-1-016013-12.

[34] Y. Li, T. Täffner, M. Bischoff, and B. Niemeyer, "Test gas generation from pure liquids: an application-oriented overview of methods in a nutshell," International Journal of Chemical Engineering, 2012, 2012: 417029-1-417029-6.

[35] D. I. Johnson and G. E. Town, "Refractive index and thermo-optic coefficient of composite polymers at 1.55 $\mu \mathrm{m}$," SPIE, 2005, 6038: 603821-1-603821-8.

[36] J. H. Schmid, M. Ibrahim, P. Cheben, J. Lapointe, S. Janz, P. J. Bock, et al., "Temperature-independent silicon subwavelength grating waveguides," Optics Letters, 2011, 36(11): 2110-2112.

[37] Y. Sun, Y. Cao, Y. Yi, L. Tian, Y. Zheng, J. Zheng, et al., "A low-power consumption MZI thermal optical switch with a graphene-assisted heating layer and air trench," RSC Advances, 2017, 7(63): 39922-39927.

[38] A. Densmore, S. Janz, R. Ma, J. H. Schmid, D. X. $\mathrm{Xu}$, A. Delâge, et al., "Compact and low power thermo-optic switch using folded silicon waveguides," Optics Express, 2009, 17(13): 10457-10465.

[39] D. Pérez, J. Fernández, R. Baños, J. D. Doménech, A. M. Sánchez, J. M. Cirera, et al., "Thermal tuners on a silicon nitride platform," ArXiv, 2016, pp. 1-13.

[40] B. X. Jing, J. Zhao, Y. Wang, X. Yi, and H. L. Duan, "Water-swelling-induced morphological instability of a supported polymethyl methacrylate thin film," Langmuir, 2010, 26(11): 7651-7655.

[41] K. Tanaka, Y. Fujii, H. Atarashi, K. I. Akabori, M.
Hino, and T. Nagamura, "Nonsolvents cause swelling at the interface with poly (methyl methacrylate) films," Langmuir, 2007, 24(1): 296-301.

[42] G. Geertz, J. Wieser, I. Alig, and G. Heinrich, "Modeling of moisture-induced stress in PMMA: a simple approach to consider sorption behavior in FEM," Polymer Engineering and Science, 2017, 57(1): 3-12.

[43] J. E. Saunders, H. Chen, C. Brauer, M. G. Clayton, W. J. Chen, J. A. Barnes, et al., "Quantitative diffusion and swelling kinetic measurements using large-angle interferometric refractometry," Soft Matter, 2015, 11(45): 8746-8757.

[44] K. Süvegh, M. Klapper, A. Domján, S. Mullins, W. Wunderlich, and A. Vértes, "Free volume distribution in monodisperse and polydisperse poly (methyl methacrylate) samples," Macromolecules, 1999, 32(2): 1147-1151.

[45] J. S. Papanu, D. W. Hess, D. S. Soane (Soong), and A. T. Bell, "Swelling of poly (methyl methacrylate) thin films in low molecular weight alcohols," Journal of Applied Polymer Science, 1990, 39(4): 803-823.

[46] J. M. Zielinski and J. L. Duda, "Predicting polymer/solvent diffusion coefficients using free-volume theory," AIChE Journal, 1992, 38(3): 405-415.

[47] S. Das and V. Jayaraman, " $\mathrm{SnO}_{2}$ : a comprehensive review on structures and gas sensors," Progress in Materials Science, 2014, 66: 112-255. 